\title{
Ecology in mind, mindful practices
}

\section{Dian Marie Hosking}

In

European Journal of Work and Organisational Psychology, 9, No 2, June 2000

Special issue: Organisational learning: relational constructionist approaches,

\author{
Guest Editors: Dian Marie Hosking \& Rene Bouwen
}

\section{Abstract}

In many areas of writing and social practice it has become commonplace to think of people and organisations as having content-specific characteristics (e.g., traits, attitudes, structures), and as conducting internal and external processes (intra and interpersonal, intra and inter-organisational...). The approach taken here is very different. Relational processes form the 'starting point', these being viewed as the medium within which social realities and learning are socially constructed. More precisely, relational processes are said to construct (a) people and things and (b) relations - as social realities. It is suggested that relations very often are constructed as being between separate and opposed entities; these being viewed as relations of 'either/or'. However, relations also may be constructed as 'both/and' - which invites a view of learning as participation by treating self and other as joined. It is this participative or ecological view that is explored here. An ecological view invites serious consideration of ways of relating that are: diverse - rather than homogenised; slow - rather than fast, and; capable of constructing deep knowledges e.g., that are less closely tied to conceptual language and to a 
disembodied sense of 'l', 'outside' of nature. Discussion briefly points to some mindful (mind-full) practices that embrace, for example, self reflecting ways, reflection on frames and questions, and reflection on conceptual ways of knowing.

\section{Some-one knows something}

Participants in western cultures usually speak of people and organisations in terms of 'ism's', talking of things (nouns as subjects and objects) and describing their characteristics using noun qualifiers (see e.g., Hosking \& Morley, 1991). So, for example, organisations (such as universities) and their contributors (leaders, managers, students...) are said to discover what knowledge 'is', and to own, teach, learn, and sell 'it'. Conventions construct an acting subject that is assumed to relate to, learn about, and produce (form, shape, structure) objects such as followers, trainees, and students. Of course, many grammars require use of both nouns and verbs, often in subject-verbobject relation. Users can try to give richness to both noun and verb forms in rather the same way, for example, that research programmes can assume and investigate both particle and wave, both stability and change. However, both/and practices are rare. Instead, verbs such as knowing and organising are treated as if they were nouns (knowledge, organisation), or they are used as verbs - but verbs capable of doing very little given the richness of the noun (adjective, pronoun...) context. So, in this "entitative" view (Hosking \& Morley, 1991), some-one with knowable, defining and stable characteristics, knows and influences some-one and/or some-thing.

\section{Constructing some-one and some-thing}

Here, primacy is given to verbs. Others have attempted this in many different ways. These include linguistically oriented approaches that advocate an operational language of 'ing' rather than 'is' (Korzybski, 1933), along with theories that emphasise humans acting - rather than personal characteristics (e.g., Sims \& Lorenzi, 1992; Weick, 1979). A relational constructionist perspective characterises the present work - a perspective that theorises the 
processes (rather than products) of social construction. This means that persons and organisations are viewed as ongoing and multiple constructions 'made in' processes, so to speak and not as the pre-existing 'makers of' processes. Premises concerning social construction processes will be outlined; more elaborated versions of these arguments are developed elsewhere (see e.g., Gergen, 1994; Dachler \& Hosking, 1995).

In a relational constructionist perspective what is and how we know it are viewed as ongoing achievements constructed in sequences of acts/events. Acts may be written or spoken but also could be gestures, artefacts, and what commonly are understood as objects in nature. In other words reality, and representation of the same through conceptual language, are not being differentiated and opposed - as is so often the case (see, for example, Gergen \& Thatchenkerry, 1996). Rather, conceptual language is understood as one of many possible ways to connect with Other (e.g., Hosking, Dachler, \& Gergen, 1995; LeShan, 1974). In addition, constructions are considered as: a mix of the already learned (conventional) and the new (emergent); ongoing, and; relational in the sense of always inter-relating connections - many of which are tacit (e.g., Polanyi, 1958; Coulter, 1989). So, what are counted as knowledge - truth, science...i.e., all social practices - can be said to be local social-historical conventions that realise and fix some sub-set from an infinity of possibilities (see e.g., Slife \& Williams, 1995; Woolgar, 1996).

\section{Constructing relations as subject-object}

The above themes have been developed over many years in many literatures including philosophy of science, interactionist social psychology, feminisms, postmodernist histories of ideas and cultures...(see e.g., Gergen, 1994 for a review). Whilst interests differ to focus e.g., on science, on gender, on nature... theorists generally have understood construction to be a process of differentiating and relating (e.g., Bateson, 1972). Further these processes have been shown to include (some sort of) differentiation and relating of self and other. So, for example, Self and Other often are differentiated and related as e.g., knower and known, observer and observed, expert and novice... 
Recent Western constructions e.g., in many science subjects - between the observer and observed, typically construct exclusive differences that are understood as opposed in an Aristotelian logic of either-or. These have been called "subject-object" constructions (e.g., Fine, 1994) where the former is viewed as active (knowing and influencing) in relation to some differentiated Other(s), treated as passive, knowable and formable, object(s) (see e.g., Berman, 1981; Hosking, Dachler, \& Gergen, 1995; Harding \& Hintikka, 1983; Plumwood, 1993).

\section{Constructing inclusive and co-operative relations}

Many have suggested and persuasively argued that subject-object relations are not enforced by the world 'as it really is'. For example, reflection on scientific practices shows that knowledge of 'the observed' cannot be entirely separated from 'the observer' (e.g., Heisenberg, see e.g., Berman, 1981; Knorr Cetina, 1981; Woolgar, 1996). Scientific practices, like other practices, may take-for-granted the separateness of Subject and Object, may strive to impose S-O relations, and may devalue deviations as bad science or as unscientific (see e.g., Harding, 1986, 1987; Slife \& Williams, 1995). However, critiques of S-O assumptions suggest that these assumptions may be regarded as relational in the sense of being constructed in relation to particular local (historically located) cultures, and in constructed in relation to particular standards (as conventions). What is interesting in the present context is that this opens-up consideration of other (non S-O) relational possibilities, how particular differentiations and relations come to be warranted, and in relation to whose and what notions of usefulness. So, for example, research need not necessarily be conducted in S-O relations (e.g., Fine, 1994; Hosking \& Ramsey, 1999), nor need relations between e.g., leader and other, or between teacher and student. What possibilities might then be afforded and constructed in ongoing relations? One possible alternative, already spoken of, is the both-and view of relations in which self and other explicitly are viewed as in inclusive, co-operative relation. This has been called a "participative world view"- where participation is understood to 
be a relational way of being and knowing and much more than a form of governance or interpersonal style (Reason, 1994; see also Addleson, this issue). Perhaps this is what others had in mind when they argued that "partnership" (rather than dominator) cultures have been constructed in which relations were constructed as different but equal rather than exclusive and opposed, right/wrong... (e.g., Eisler, 1987). The possible power of such arguments begins to be felt when they are understood to be about a participative way of being, and not about liberal ideology in the context of science, exclusive relations, and universal rights and wrongs.

\section{Nature in mind and mind in nature}

An inclusive view of relations seems very radical indeed when considered in relation to an S-O construction such as is embraced by the Cartesian construction of mind separate from internal and external nature ( $\mathrm{S}$ or $\mathrm{O}$ ). The latter is a defining assumption of what Sampson called "possessive individualism" (Sampson, 1993) - a world view in which mind and other personal characteristics are differentiated (set apart), nominalised (made into nouns), and "spatialized" (viewed as something, in some space, e.g., Jaynes, 1976). In the Subject-Object view $S$ is 'outside' of and acts over $O$ (internal and external nature) to make the latter in some way serviceable to the subject as a provider of natural or human resources. In contrast, the "participative" or co-operative view of relations can be thought of as a construction in which $\mathrm{S}$ and $O$ are enfolded and co-dependent as in the Yin/Yang symbol. An inclusive view of relations as co-operations is an eco-logical view. It offers an "ecological construction of mind" (Bateson, 1972) - or indeed of all relational processes. Minding becomes understood as a world of pattern, of form in process (relating), and minding other (e.g., 'nature') is minding self.

Constructionist arguments offer no firm foundation for the claim that either inclusive or exclusive ways of relating are more true or better in any universal, transcendental sense. Indeed, persuasive arguments could be made for the pragmatic value of many S-O ways of relating. This said, it is increasingly argued that the pragmatics of particular local cultural/historical considerations 
may call for more inclusive ways of relating. For example, new world-wide communication technologies, globalisation, increasing inequalities in financial wealth and economic infrastructure, destruction of forests, landscapes and communities, pollution... raise issues that do not seem tractable through more subject-object ways of relating. A changed epistemology - changed forms of practice - seem needed (e.g., Bateson, 1972; Reason, 1994; Dachler \& Hosking, 1995). Again, Reason puts the point well, arguing (quoting Mumford) for the need to "fashion a myth" that might be "of service to our time"(Reason, 1994, p.16). This might be a "participative world view" that constructs mind in nature and nature in mind.

\section{How can we know?}

Relating has been theorised here as the co-ordination of events. It was further suggested that these may be constructed in anything and everything that might commonly be understood as verbal and non-verbal, as talk and other kinds of action, and as artefacts and as facts of nature (e.g., LeShan, 1974). In their theoretical work writers sometimes speak of text - context relations perhaps, for certain purposes, too suggestive of conceptual language - and sometimes of act - supplement relations, perhaps understating the involvement of what usually would be understood as natural and artificial/made objects (see Hosking, Dachler, \& Gergen, 1995). Shifting to a view that joins conceptual language with other forms of text and that decenters humans as 'knowers and shapers' in subject-object relations invites and legitimates changed forms of praxis and therefore new constructions of learning.

Learning, when eco-logically constructed, could involve less emphasis on conceptual language - perhaps desirable given the limits that conceptual language tools construct and reconstruct (e.g., Reason, 1994; Bass \& Hosking, 1999). Ecological ways of knowing include ways that are embodied, enchanted, sensual, analogical... joined with the landscapes of our being (see e.g., Castaneda, 1974; Eliade, 1964; Heron, 1992; Reason, 1993; Walck, 1996). Further, shifting attention to relational construction processes is a shift 
towards the 'how' of knowing, relative to knowledge that i.e., propositional knowledge. Finally, and of central interest here, is the invitation to view knowledge and the criteria by which it is evaluated and certified as true, relevant, useful as multiple, local, and contestable rather than singular, general/universal, and independent of power considerations (e.g., Gergen, 1994). Our arguments about social construction processes have major implications for education and training. This is especially true given arguments about eco-logical ways of relating - arguments that now enfold (or rather do not separate) individual and organisational learning.

\section{Change in mind; some possibilities for learning}

These relational - constructionist arguments do not provide universal or fixed warrants for any particular constructions (as content) or ways of relating. Put slightly differently, it is a mistake to think of a particular method - of research, change, teaching or learning... as constructionist (whilst others are not) or as necessarily more fitting or more desirable than others - in relation to constructionist premises. However, many ways of knowing (learning, changing, 'going on'...) that were deemed irrelevant, fallible, or foolish in relation to entitative, subject-object constructions, now achieve warrant and as yet unimagined possibilities are opened up; we shall discuss just a few.

\section{Self reflecting ways}

Relational processes have been argued to implicate the ongoing construction of self, other(s), and relationships. One implication is that participants might reflect on their ongoing (re)constructions and how they achieve them. For example, teacher and learner, researcher and researched, change agent and client often are constructed in relations where the former acts as the knowing expert who has stocks of knowledge/expertise they can apply to form the learner, research process, or intervention in a one-way relationship. However, such subject-object co-ordinations may not always be helpful. For example, lecturers may grumble that their students lack initiative, do nothing but regurgitate what they are given, do so in an uncritical fashion and so on. Similarly, the client in therapy or organisation in 'development' might become dependent - a difficulty commonly constructed by practitioners - and might not learn how to learn for them selves. Constructionist themes suggest that these co-ordinations might be viewed as the learned reproduction of subject-object relations. Again, relational constructionist arguments suggest that - in addition to what ever else might be learned about self and/or other - participants' learn a way of 
relating - one that might not be helpful. Indeed, perhaps the recent fashions for empowering and for organisational learning are indicative of the limits of subjectobject ways of relating. Such fashions are more of the same unless they change the limits imposed by subject-object relations.

Constructing different but equal (not S-O) relations has been shown to be possible and helpful in relation to particular pragmatic projects. There is much work to be done to develop ways of constructing relations of this sort; perhaps at present it is easiest to say what to avoid, what not to do (e.g., Burr, 1994; Gergen \& Thatchenkerry, 1996). However, as will be explored shortly, it is increasingly possible to identify relations of this kind being constructed in family therapy and organisation development.

\section{Reflecting frames}

Many have drawn on the work of Bateson, Watzlawick and others to argue the value of reframing for learning and change. For example, Reason (1994) speaks of the importance of a "reframing mind" reflecting on the frameworks constructed in language including presuppositions that hold particular constructions/practices in place. Other writers emphasise action and act-supplement co-ordinations as they frame and reframe, particularly co-ordinations that are not linguistically expressed (e.g., Bass \& Hosking, 1998; Hosking \& Bass, 1999).

Constructionist arguments concerning the significance of frames also lend increased importance to careful theoretical work. This is because that which 'the standard view of science' calls method becomes broadly defined to include theoretical and pre-theoretical presuppositions (implicits), including assumptions about self-other relations, along with the questions so invited; theory and method are thoroughly intertwined.

Deconstruction also is given a changed role and importance. Deconstruction makes it possible to learn the constraining contributions of language tools, taken-for-granteds, and methods - and shows that all methods 'find' what they are "tuned for" (e.g., Slife \& Williams, 1995). Deconstruction can be a particularly instructive way of working with, for example, students, clients in therapy, or groups in organisation development to identify e.g., taken-forgranteds that hold a particular 'game' or world view in place (see e.g., Isaacs, 1993; Barrett, Thomas, \& Hocevar, 1995). Learning the importance of frames, deconstructing and reconstructing - explicitly or not - may open-up a world of yet to be imagined, yet to be created possibilities. So, for example, it becomes 
possible to see learning - including what traditionally has been called research, or studying, or organisation development - as a process (re)constructing subject-object co-ordinations (S acting from the 'outside', over O). Equally, it becomes possible to learn in dialectical 'insider' or co-operative relations ("knowing from within", Shotter, 1993) between different but equal self and other.

Reflecting and reframing can be done by shaking notions of defining characteristics and opposites, by working 'in between', with edges, so to speak. Some have spoken of constructing the "liminal" - where 'what is' is experienced as multiple possibilities teetering on so many ledges. Turner locates the liminal where "the past is momentarily negated, suspended, or abrogated, and the future has not yet begun, an instant of pure potentiality when everything, as it were, trembles in the balance" (Turner, 1982, p.44; in Barry, 1997). This is very different from the over-generalised, and recipeoriented approaches increasingly characteristic of business school education; it is very different from knowledge management approaches that entify and fix knowledge whilst neglecting processes, possibilities and power.

'Messing about' with frames can be done in many ways. These include: playing the fool, mask work, and re-sculpting particular happenings; shaking up master scripts, or narratives e.g., by working with multiple narratives or by re-storying, and; constructing scenarios in which its possible to reflect on the many different constructions that can be made of 'the same thing' (e.g., Barry, 1997; Smircich \& Stubbart, 1985; Farrelly \& Brandsma, 1974). One potentially powerful method is "public reflection" in which different persons and group voice their different constructions for other to hear and e.g., dialogue with (see e.g., Anderson, 1990). Related methods involve voicing multiple constructions - where participants have a chance to hear how very differently others have constructed something and/or someone. The present author has used simulations and case studies to work in these ways e.g., with students working with how a particular construction works. Lecturers, trainers, consultants... do not need to impose their 'right answer' or to seek consensus on problems and changes. Instead, they may use a variety of means to join with others to reflect on differences in: accounts; assumptions; what's 
identified as a text (and what ignored); patterns of relations and the possibility of a "difference that makes a difference" (Bateson, 1972).

Reflecting questions

It was earlier proposed that theory and data can be viewed as intermingled and questions may be viewed as theory laden, reflecting local-cultural textcontext relations. Questions, reflecting particular taken-for-granteds and pragmatic projects, constrain possible co-ordinations. Of course, this makes perfect sense when their job is to 'find out' what is (was, will be) in order e.g., to identify what happened and who is to blame... However, the "sense" is not so obviously "perfect" once we view questions as literally (re)constructing the world we know, ourselves, and our relations and not so much as finding out (about some pre-existing reality).

Interviews can be used to illustrate some of the ways that question-answer co-ordinations construct realities, including the realities of some relationship therapist-client, selector- job applicant, researcher and research objects. Consider the different kinds of relations that might be constructed depending e.g., on who asks questions and who answers, who decides on the relevant and acceptable constructs, whose implicit assumptions most structure the interaction and decide what's important and what counts as an acceptable answer... Again, there is the possibility that ways of relating, whatever else they do, construct a Subject who is active, knowing, healing, teaching, developing ... in relation to Other who co-ordinates with these questions as a knowable and formable object. Readers who find these arguments persuasive might be ready to warrant the notion that these interactions (re)constructed the identities and relations of participants just as much as they 'found out' about them.

Should different but equal relations and multiple knowledges be valued, how might this be done? Various so-called postmodern approaches e.g., to therapy, to 'individual' change work, and to organisation development (OD) provide considerable help. For example, it seems that questions might usefully be asked out of a not knowing stance e.g., to minimise constraints - to be open to new possibilities and to multiple realities - to negotiate locally rather than impose 'outsider' constructions (e.g., Anderson, 1997; Janov, 
1995). In addition, Socratic dialogue (see e.g., Slife \& Williams, 1995) may be less likely to reproduce existing assumptions and conventions. One obvious example would be the practices that seem to assume a knowing teacher, a ready to know student, transferable conceptual knowledge etcetera. Socratic dialogue may be employed to reflect on frames, and offers the possibility for multiple voices and new questions.

Everyday praxis constructing multiple knowledges

These relational constructionist arguments mean that everyday practices construct local ways of knowing and that which is to be known. Consider, for example, activities usually thought of as data collection, as 'finding out', or research. Rather than viewing the research process as what mediates between theory (input) and data (output) it can be treated as interesting in its own right - both as a vehicle for knowing and that which is to be understood. Just as the meaning and importance of method were re-contextualised in relation to constructionist narratives, the same may be done with abstract theory and findings (see e.g., Gergen, 1994). To do so puts in question those teaching, training, and consulting practices that offer abstract theories and frameworks and generalisable findings as that which must be learned. Again, this often is the case in business schools, in standardised training packages, and in consulting that depends on generalising content from one context to another.

It was earlier suggested that any praxis can be constructed in monophonic, subject-object relations or in polyphony where multiple knowledges may be constructed simultaneously in relations of different but equal. It is perhaps worthwhile pausing to emphasise that the sense some one makes of this depends on their 'world view', paradigm, or perspective. In the present variant of constructionism, talk of multiple knowledges does not refer to different constructions of 'the same thing' i.e., to multiple subjectivities as variants around objective knowledge. Rather multiple knowledges are theorised as different local 'fixings' of different patterns of possibilities where each fixing affords participants different ways of being and different possible supplements in ongoing relations. Further, such differences can perfectly well co-exist, despite the common notion that e.g., shared notions of reality or at least 
shared goals are necessary for co-ordinated action. For example, lecturer and students may construct many different and perhaps conflicting narratives about a lecture but these do not necessarily get co-ordinated. Instead the lecturer may very likely talk and enough students will co-ordinate by staying quiet i.e., will stay within conventional limits of what is an acceptable coordination, such that the lecture can go on.

To continue with this point, it may or may not seem obvious that multiple knowledges can co-exist in a marriage, in therapy, and in organisational life without any intentional 'fixing' e.g., to agree or impose one version of reality. Indeed, there may be many pragmatic standards in relation to which not voicing multiple knowledges in some sense 'works'. For example, experienced negotiators know that it often is wise not to raise a divisive issue that could not be resolved. This said, imposing one dominant voice may 'work' in relation to some reality constructions or local fixings. For example, when considered from some points of view (and perhaps not others) it might 'work' to impose and attempt to 'fix' some organisation-wide mission, or the framing of some activity as 'research'. Indeed, the construction that things are 'not working' is often accompanied by attempts to 'remove' differences. However, it seems increasingly evident that "homogenised knowledge" and knowledge that is "fast" (Orr, 1997) may have disastrous consequences, more quickly and more extensively than ever before - before globalisation, the web, agri-business, the dominance of marketing, world-wide travel...

Deep knowledge and patterns

These arguments have reflected on the common and conventional separations of theory and practice and on the heavy reliance (by some) on conceptual language as a tool for representing knowledge. With respect to the theory-practice relation, these relational arguments make way for many local (indigenous) experts and locally grounded knowledges. This, in turn, may make the space for diverse gender and ethnic voices to participate in different but equal relations, constructing locally meaningful ways of knowing and local definitions of development (e.g., Abell \& Simons, this issue). This is true whether we are thinking of e.g., individual, organisational, or community development. 
These reflections on conceptual knowledge suggest that social constructionism too often gets absorbed with words, with grammars, with linguistically expressed metaphors, and written narratives... Conceptual language, like all ways of relating, constructs limits. The pattern that connects (Bateson, 1972) does so not only in language but includes ways of participating that reclaim the body (see Reason, 1994) e.g., through physical disciplines like Tai Chi, connected breathing, and meditation. These methods also may allow experience of the world unmediated by language, unmediated by a sense of 'l'. Such methods may allow participation in what some speak of as non-ordinary realities (e.g., LeShan, 1974; Castaneda, 1974).

Slow knowledge, future participation

The present arguments have joined with those who have suggested that there might be might be a "myth for our times" that could be more useful - in relation to certain projects - than the entitative myth. This would be to put to one side the entitative narrative of subject-object relations, its reliance and emphasis on language as representation, and its centring of a singular, and in some degree knowable, real world. Writers have used terms such as "future participation" (Reason, 1994) and "slow knowledge" (Orr, 1997) to speak of many relevant themes. This said, relational constructionist arguments also suggest it is wise to avoid becoming too attached to any paradigm (Bateson, 1972; Bandler \& Grinder, 1975; Berman, 1981). Knowing/acting slowly, participatively, involves living with possibilities rather than certainty; including multiple voices - including voices without words; taking care of patterns, constructing forms of practice with other in mind. Perhaps learning - be it in the context of education, training, development or change - will offer most future possibilities when inclusive, slow, and mindful. 